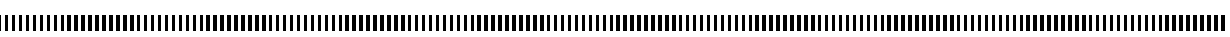

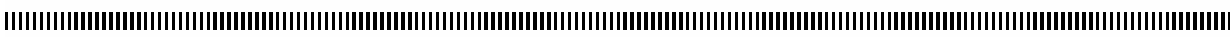

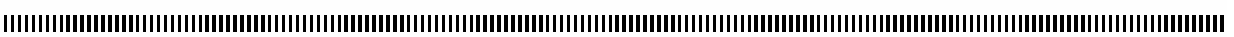

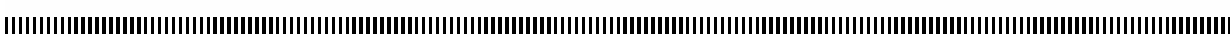

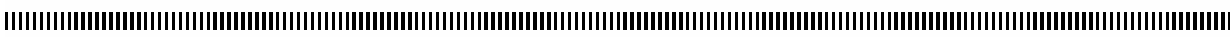

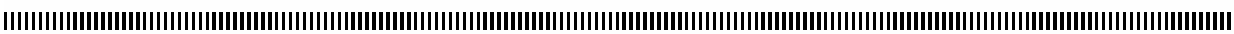

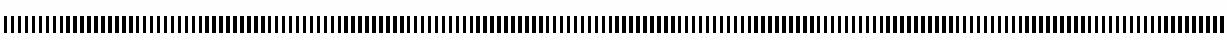

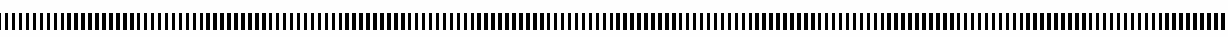

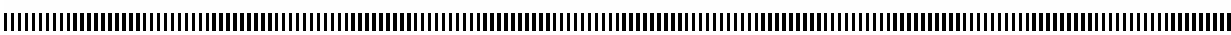

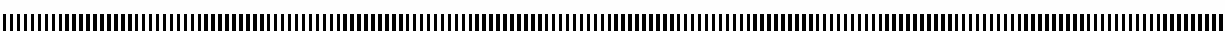

\title{
A dynamical model of plant growth with full retroaction between organogenesis and photosynthesis
}

\author{
A. Rostand-Mathieu*, P.-H. Cournède*, P. de Reffye** \\ *Laboratoire de Mathématiques Appliquées aux Systèmes, Ecole Centrale Paris \\ FRANCE \\ mathieu@mas.ecp.fr \\ **Laboratoire AMAP, CIRAD \& INRIA \\ FRANCE
}

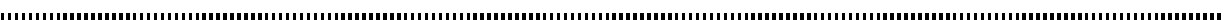

RÉSUMÉ. Dans ce papier sont présentés de nouveaux développements mathématiques en modélisation et simulation de la croissance des plantes. GreenLab est un modèle structurefonction, qui combine l'organogenèse (architecture) et la photosynthèse (production et répartition de biomasse). La rétroaction entre la photosynthèse et l'organogenèse est introduite ici. Nous présentons l'influence de la biomasse disponible sur le nombre de métamères par unité de croissance et sur le démarrage des bourgeons. La théorie est ensuite appliquée à des modèles d'arbre simples, dont on étudie divers comportements.

ABSTRACT. In this paper are presented new mathematical developments in plant growth modelling and simulation. GreenLab Model is a functional-structural plant growth model, it combines both organogenesis (architecture) and photosynthesis (biomass production and repartition). New improvements concern the retroaction of photosynthesis on organogenesis. We present in this paper the influence of available biomass on the number of metamers in a growth unit and on the branching. The general theory is introduced and applied to simple trees. Some interesting behaviours are underlined.

MOTS-CLÉS : modélisation de croissance des plantes, simulation, système dynamique, modèle structure-fonction.

KEYWORDS: plant growth modeling, simulation, dynamical system, functional-structural model.

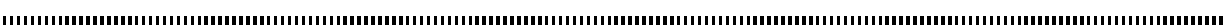

Volume 4-2006, pages 101 à 107 - A R I M A

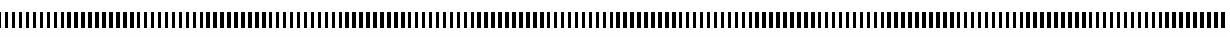

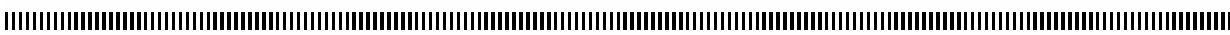

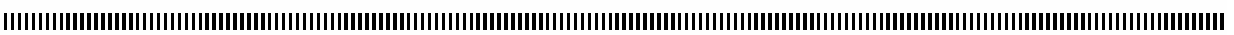

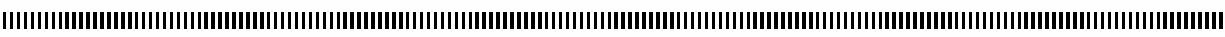

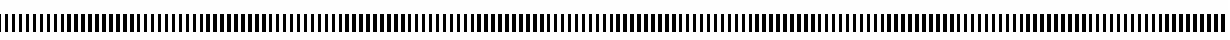
|

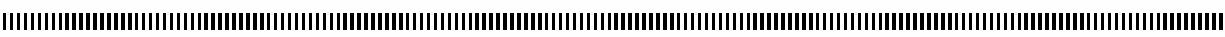

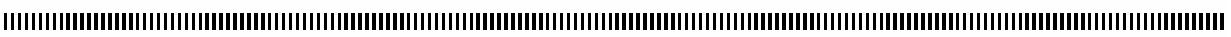

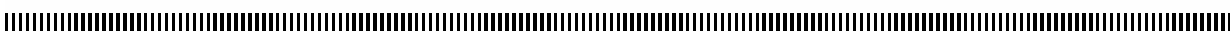
| 


\section{Introduction}

Functional-Structural Models ([7]) aim at controlling all sides of plant growth by combining organogenesis and photosynthesis. So far, few models exist with their corresponding simulation codes ([2],[3],[5]). Recently, Yan et al., see [4], proposed a functional-structural model in the form of a dynamical system, GreenLab, and based on simple relevant choices from both biological and mathematical point of views. The mathematical formalism introduced allows to implement optimization and control techniques for agronomic applications.

In the previous version of the model, the organogenesis was determined by a deterministic or stochastic automaton, without any influence of the photosynthesis. In this paper, the complete feedback between photosynthesis and organogenesis is introduced: a variable number of organs will be constructed according to the ratio between the biomass supply provided by the photosynthesis and the plant demand. We start by giving a new form for the growth cycle taking into account for the first time the bud existence. Then, we introduce the influence of the retroaction on two kinds of organs: first, the number of metamers per growth unit, secondly, the branching pattern. Lastly, for simple cases, we study the behaviour of the system, from theoretical and numerical point of views.

\section{Description of the model}

Plants are supposed to grow in cycles. Organogenesis and photosynthesis are computed simultaneously in a recurrent loop of growth. After describing a cycle, we detail the mathematical formalism used to represent the plant.

\subsection{Progress of a growth cycle}

From an architectural point of view, the plant is decomposed in basic elements and simulated by an automaton, whose time step is a growth cycle:

- a metamer is a set of organs made of leaves, internodes, buds, fruits.

- a growth unit, noted GU, is an elementary portion of an axis. It is a succession of metamers set in place during the same growth cycle. It is supposed to be completely preformed in a bud (no neoformation possible).

An organ is characterized by a chronological age (number of growth cycles since its appearance) and a physiological age (type of bearing axis [1]) varying from 1 to $P_{m}$. 
In the photosynthesis model, the plant constructs its first organs from the seed. Then, during a cycle, the leaves make photosynthesis to produce biomass by utilizing carbon dioxide, light energy, and water. We introduced here the notion of bud to model the retroaction and determine the number of new organs: first, if there is not enough matter, some buds may die (which used to be probabilistic) and then, the number of preformed organs in a functional bud is computed with the matter allocated to it: what goes to a bud is proportional to its sink value and to the pool of biomass reserves divided by the total of the buds sinks, called the buds demand. The bud blossoms at the beginning of the next cycle, and so on. We can sum up this functioning in the following 6 steps:

1. Calculation of the available biomass fabricated by the leaves during the cycle.

2. Calculation of the buds demand, considering all the potential buds.

3. Determination of the number of functional buds, according to the ratio between biomass and demand, calculation of their demand.

4. For each bud, computation of the preformed growth unit: calculation of the number of metamers according to the ratio between biomass and demand, and partitioning of the fresh matter between the future organs.

6. Blossoming of the buds at the beginning of cycle $n+1$

For the sake of clarity, we suppose that the organs grow and function during only one cycle. This is the case of most temperate trees. Also secondary growth is neglected (no layers), and so is the root system. However, the model can easily be extended.

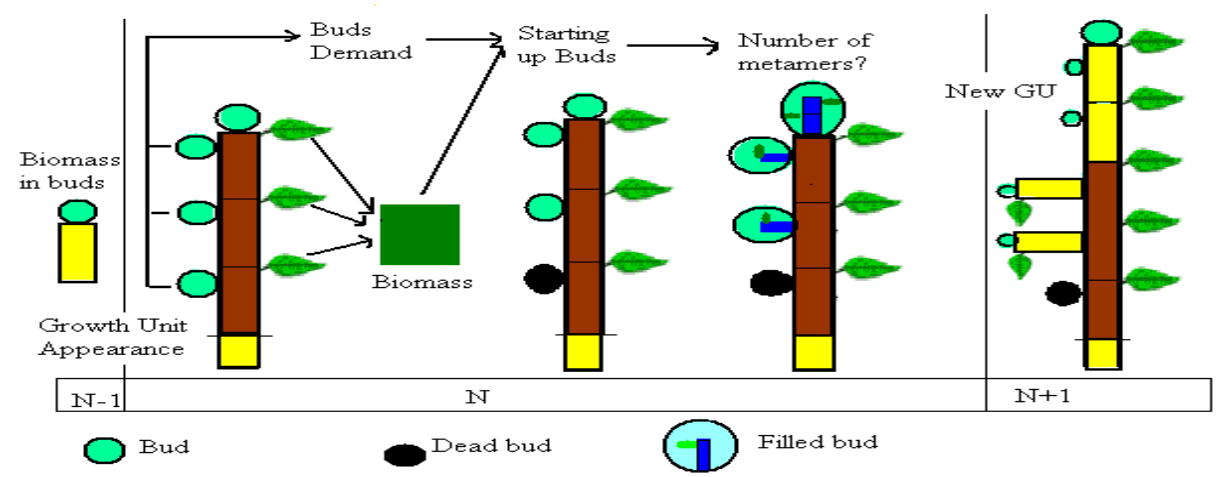

Figure 1. Cycle progress

\subsection{Mathematical formalism}

In this section, we introduce the formalism of the mathematical model. A more achieved form related to Automatic Control theory is presented in [6]. 


\subsubsection{Notations}

A bud of physiological age $\mathrm{k}$, bud(k), gives birth to a growth unit of physiological age $\mathrm{k}, \mathrm{GU}(\mathrm{k})$, that is a succession of metamers $(\mathrm{k}, \mathrm{j})$, each of them bearing $M_{k, j}$ potential axillary buds $(\mathrm{j})$ and $n_{k, j}^{a}$ leaves. We note $u_{k, j}^{n}$ the number of metamers $(\mathrm{k}, \mathrm{j})$ in $\mathrm{GU}(\mathrm{k}), \mathrm{j}$ varying from $\mathrm{k}$ to $\mathrm{P}_{\mathrm{m}}$.

$N_{k}^{o, n}$ is the number of botanical elements o created at the beginning of cycle n, $p^{o}$ their sinks (a for leaf, e for internode, $m$ for metamer, $b$ for bud, UC for growth unit). D stands for a demand and $Q^{n}$ is the biomass produced at growth cycle n.

$N^{f b, n-1}$ functional buds(fb) are in the tree at the end of cycle n-1. They blossom at the beginning of cycle n, so $N_{k}^{f b, n-1}=N_{k}^{U C, n}$. Therefore there are $N_{k}^{U C, n} u_{k, j}^{n}$ metamers(k,j) in the tree and we deduce the number of leaves, internodes...

Finally, to describe the plant at the beginning of cycle $\mathrm{n}$, we use the variables $X^{n}=\left[\left[N_{k}^{U C, n}\right]_{k=1 . . P_{m}} ;\left[u_{k, j}^{n}\right]_{k, j=1 . . P_{m}}\right]$ and $S^{n}=\left[S_{k, j}^{n}\right]_{k, j=1 . . P_{m}}$, array of the surface areas of leaves. We note $\mathrm{A}, \mathrm{B}$ and $\mathrm{E}$ arrays representing respectively plant construction rules, relations between sinks and sources, and environmental parameters.

\subsubsection{Photosynthesis}

In the photosynthesis model, each leaf produces matter $q^{n}$ according to an empirical function depending on its surface $S^{n}$, on $r 1, r 2$ hidden parameters to assess. We suppose that only the one-cycle old leaves work to fill the biomass reserve. We have:

$$
\begin{aligned}
& q^{n}=\frac{E}{\frac{r_{1}}{S^{n}}+r_{2}}, \quad Q^{n}=\sum_{k=1}^{P_{m}} N_{k}^{U C, n} \sum_{j=k}^{P_{m}+1} \frac{n_{k, j}^{a} u_{k, j}^{n} E}{\frac{r_{1}}{S_{k, j}^{n}}+r_{2}}=Q\left(X^{n}, S^{n}, E, B, A\right) \\
& \text { 2.2.3 Organogenesis }
\end{aligned}
$$

\subsubsection{Organogenesis}

\subsubsection{Buds modelling}

In the tree, there are $N_{k}^{p b, n}$ potential quds of physiological age $\mathrm{k}(3)$ :

$$
N_{k}^{p b, n}=\sum_{j=1}^{k} N_{j}^{U C, n}\left(u_{j, k}^{n} M_{j, k}\right)+N_{k}^{U C, n}
$$

and their demand is (4) :

$$
D^{p b, n}=\sum_{k=1}^{P_{m}} N_{k}^{p b, n} p_{k}^{b}
$$

According to the available biomass, some buds may die. Their behaviour depend on their characteristics (physiological age) but not necessarily on the branching order in the 
tree. We consider for the moment that terminal buds always keep functioning. In each $\mathrm{GU}(\mathrm{k})$, we determine the number $m_{k, j}^{n}$ of functional buds among the $u_{k, j}^{n} M_{k, j}$ identical potential ones, and set them in place in the growth unit according to botanical laws.

$$
m_{k, j}^{n}=G_{k, j}\left(u_{k, j}^{n} M_{k, j} ; p_{j}^{b} \frac{Q^{n}}{D^{p b, n}}\right)
$$

Hereafter, we compute the total number of functional buds and their demand:

$$
N_{k}^{f b, n}=\sum_{j=1}^{k} N_{j}^{U C, n} m_{j, k}^{n}+N_{k}^{U C, n} \quad \text { and } \quad D^{f b, n}=\sum_{k=1}^{P_{m}} N_{k}^{f b, n} p_{k}^{b}
$$

as $N_{k}^{U C, n+1}=N_{k}^{f b, n}$, finally:

$\left[N_{k}^{U C, n+1}\right]_{k=1 . . P_{m}}=F_{1}\left(\left[N_{k}^{U C, n}\right]_{k=1 . . P_{m}},\left[u_{k, j}^{n}\right]_{k, j=1 . . P_{m}},\left[S_{k, j}^{n}\right]_{k, j=1 . . P_{m}} A, B, E\right)$

\subsubsection{Metamers creation}

Each bud(k) receives an amount of matter proportional to its sink, $q_{k}^{b, n}(8)$ which determines the number of metamers $(\mathrm{k}, \mathrm{j})$ that it will create $u_{k, j}^{n+1}(9)$ :

$$
q_{k}^{b, n}=p_{k}^{b} \frac{Q^{n}}{D^{f b, n}}, \quad u_{k, j}^{n+1}=f_{k, j}\left(q_{k}^{b, n}\right)=F_{2}\left(\left[N_{k}^{U C, n}\right]_{k=1 . . P_{m}}, A, B, Q\right)
$$

We note $d_{j}^{b, n}$ the organs demand inside the bud and each one receives $q_{j, l}^{n+1, o}$. They appear at the beginning of cycle $\mathrm{n}+1$, their volumes being computed by allometry rules.

$$
q_{j, l}^{n+1, o}=\frac{p_{j, l}^{o} q_{j}^{b, n}}{d_{j}^{b, n}}, \quad \text { with } \quad d_{j}^{b, n}=\sum_{p=j}^{P_{m}} u_{j, p}^{n+1} p_{j, p}^{m}
$$

In particular, leaf area is sil1, $^{-1}$

$$
S_{j, l}^{n+1}=\frac{q_{j, l}^{n+1, a}}{e}=H_{j, l}\left(\left[N_{k}^{U C, n+1}\right]_{k=1 . . P_{m}},\left[u_{k, j}^{n+1}\right]_{k, j=1 . . P_{m}},\left[S_{k, j}^{n}\right]_{k, j=1 . . P_{m}}, B\right)
$$

\subsubsection{Dynamical system}

By combining the above organogenesis and photosynthesis equations, we can obtain the following dynamical system describing the plant growth:

$$
\left\{\begin{array}{l}
S^{n+1}=H\left(X^{n}, S^{n}, A, B, E\right) \\
X^{n+1}=F\left(X^{n}, S^{n}, A, B, E\right)
\end{array}\right.
$$




\section{Results}

In this section, we present some theoretical and numerical examples for the variable number of metamers per growth unit and the variable number of axillary branches. The simulation results are obtained with the $\mathrm{C}++$ software Digiplant developed in ECP.

\subsection{Variable number of metamers per GU in young seedlings}

In nature, tree seedlings have growth units with few short metamers. Progressively, growth unit size increases. Here we study a young unbranched seedling. At each cycle, its terminal bud gives birth to a new growth unit, whose number of metamers varies. Growth equations (6)-(11) become:

$$
\begin{aligned}
& u^{n+1}=f\left(Q^{n}\right), \text { with } Q^{n}=\frac{u^{n} E Q^{n-1}}{\chi u^{n}+\beta Q^{n-1}} \text { and } \chi=\frac{r_{1} e p^{m}}{p^{a} p^{b}}, \beta=r_{2} \\
& N^{U C, n+1}=1, \quad S^{n+1}=\frac{p^{a}}{e} \frac{Q^{n}}{p^{m} u^{n+1}},
\end{aligned}
$$

We choose $f: x \rightarrow C\left\langle x^{\alpha}\right\rangle$, where $\langle x\rangle$ is the rounded value of $\mathrm{x}$.

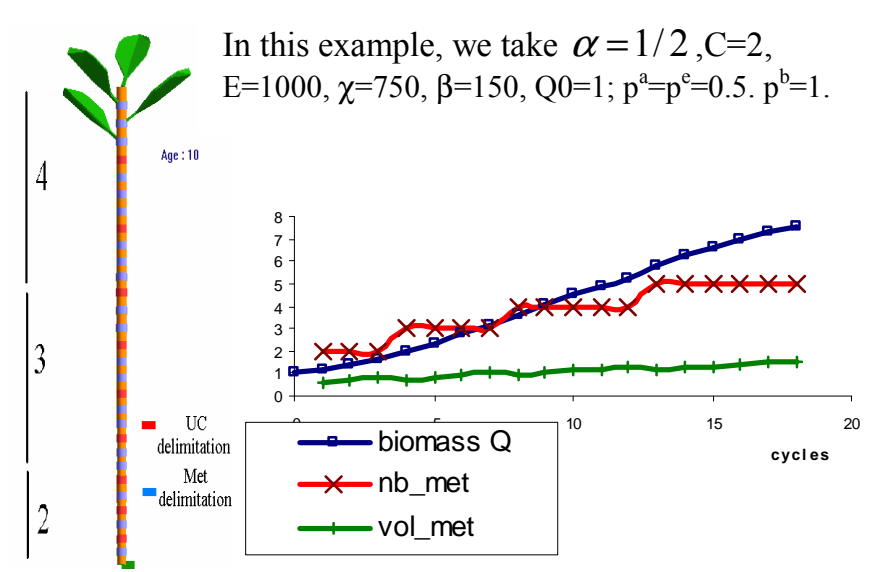

\begin{tabular}{|cccc|}
\hline $\mathrm{t}$ & $\mathrm{Q}^{\mathrm{n}}$ & $\mathrm{u}^{\mathrm{n}}$ & $\mathrm{Q}_{\text {met }}$ \\
\hline 0 & 1,00 & 0 & 0,00 \\
1 & 1,21 & 2 & 0,61 \\
2 & 1,44 & 2 & 0,72 \\
3 & 1,68 & 2 & 0,84 \\
4 & 2,01 & 3 & 0,67 \\
5 & 2,37 & 3 & 0,79 \\
6 & 2,73 & 3 & 0,91 \\
7 & 3,08 & 3 & 1,03 \\
8 & 3,56 & 4 & 0,89 \\
9 & 4,02 & 4 & 1,01 \\
10 & 4,47 & 4 & 1,12 \\
\hline
\end{tabular}

If $\alpha>0$, the biomass and the number of metamers have a limit that can be easily computed from the recurrent equations (13). This behaviour is quite similar to that of young beech tree. 
Some interesting behaviours frequently observed on real plants (rythms, chaos ...) are obtained for some particular value of $\alpha$.
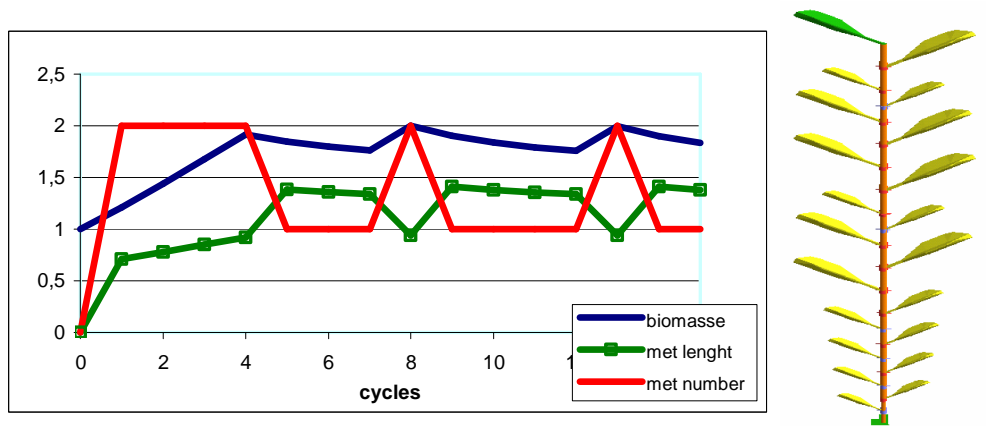

Example with $\alpha=-0.5$

We notice that, after a while, the series becomes periodical $Q^{n+5}=Q^{n}$

\subsection{Functional buds in a Roux model}

We study a tree with a trunk and one type of branches (2 physiological ages). Growth units are all composed of one metamer, bearing $\mathrm{m}$ leaves. Metamers on branches have no axillary bud. The terminal bud always functions but the lateral ones may not:

$$
\begin{aligned}
& \left\{\begin{array}{l}
N^{U C, n+1}=G\left(N^{U C, n}, \frac{Q^{n}}{D^{p b, n}}\right)=N^{U C, n}+\left\langle 0.435 \frac{Q^{n}}{m+N^{U C, n}}\right\rangle, u^{n+1}=1 \\
S^{n+1}=\frac{1}{e(m+1)} \frac{Q^{n}}{D^{j b, n}} \quad \text { with } \quad Q^{n}=\frac{m N^{U C, n} E Q^{n-1}}{\frac{r_{1} e}{p^{a}} N^{U C, n}+r_{2} Q^{n-1}}=h_{n}\left(Q^{n-1}\right)
\end{array}\right. \\
& A=\frac{r_{1} e}{p^{a}}, \mathrm{E}=1000, \mathrm{e}=0.05, \mathrm{r} 1=7500, \mathrm{r} 2=75,2 p^{a}=p^{e}=0,5, p^{b}=1, m=2
\end{aligned}
$$

We can deduce the conditions satisfied by the first cycle of branching $l$ :

$$
h_{1}^{l-2}\left(Q^{0}\right)<(m+1) 0.5 / 0.435 \leq h_{1}^{l-1}\left(Q^{0}\right),
$$

and finally $l=8$ here (that is $m_{1,2}^{7}=1$ ).

After some time, two branches appear at each cycle. Then, the metamer volume tends to a limit $q_{\text {l }}$ that is independent of the choice of the function $\mathrm{G}$, and exists even with a linear unbounded function:

$$
q_{l}=\frac{m E-A}{r_{2}}=6.67
$$




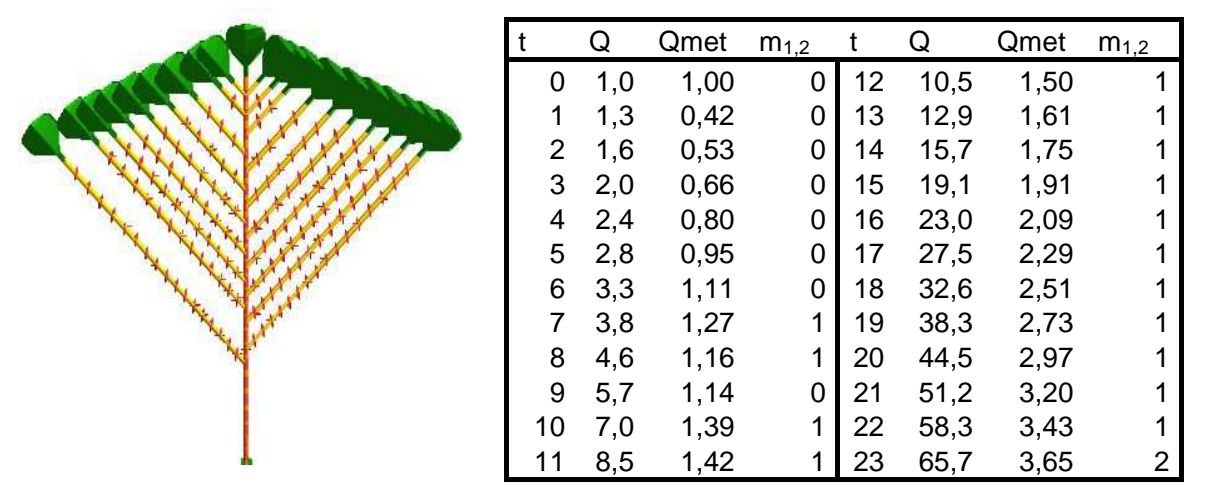

\section{Conclusion}

We have presented the bases of a theoretical model describing the influence of photosynthesis on the number of metamers per growth unit and on branching. The model can be easily extended to more complex cases. However, it needs to be fitted to real plants, which raise a problem of choice and adjustment of its parameters. Therefore, complementary studies (implying confrontation of the model to real plants, like beech tree and coffee tree) are in process in order to refine the parameters definition.

\section{Bibliography}

[1] Barthélémy D., Caraglio Y., Costes E.,1997, Architecture, gradients morphogénétiques et âges physiologiques chez les végétaux, in Modélisation et simulation de l'architecture des végétaux, Sciences Update, Editions INRA : 89-136.

[2] Jallas E., Sequeira R., Martin P., Cretenet M., Turner S., and McKinion J., 1999, Virtual cottons, the firstborn of the next generation of simulation model, in Beltwide Cotton Conferences, Orlando, Florida, pp. 393-395.

[3] Perttunen J., Sievänen R., Nikinmaa E., Salminen H., Saarenmaa H., Väkevä J., 1996. LIGNUM: a tree model based on simple structural units, Annals of Botany 77. 87-98.

[4] Yan H.-P., Kang M.-Z., de Reffye P., Dingkuhn M., 2004, A Dynamic, Architectural Plant Model Simulating Resource-dependent Growth, in. Annals Of Botany 93: 591-602.

[5] de Reffye P, Fourcaud T, Blaise F., Barthélémy D., Houllier F., 1996. An Ecophysiological Model for Tree Growth and Tree Architecture, in: Silva Fennica eds., Workshop on Functional Structural Tree Models. Helsinki.

[6] de Reffye P., Goursat M., Quadrat J.P. and Hu Bao Gang, 2003. The Dynamic equations of the tree morphogenesis GreenLab model. In: PMA'03, Beijing.

[7] Sievänen R., Nikinmaa E., Nygren P., Ozier-Lafontaine H., Perttunen J., Hakula H., 2000, Components of a functional-structural tree model, Ann. For. Sci. 57, 399-412. 